\title{
Implementation of a Progressive Three-Year Point of Care Ultrasound Curriculum for Internal Medicine Residents
}

Kim Lewis BSc, BEd, MSc, MD, Leslie Martin, Adam Mazzetti, Abubaker Khalifa, Karen Geukers, Matthew Sibbald, Andrew Gibson, Zahira Khalid, Lori Whitehead, Khalid Azzam

DOI: 10.22374/cjgim.v13i1.222

\section{About the Authors}

Dr. Kimberley Lewis is a 4th year fellow in Critical Care at McMaster University. Dr. Leslie Martin is a MHPE Candidate through Maastricht University and a Clinical Scholar within the Department of General Internal Medicine at McMaster University. Dr. Adam Mazzetti is a 4th year fellow in General Internal Medicine at McMaster University. Dr. Abubaker Kalifa is a 5th year fellow in Critical Care at McMaster University. Dr. Karen Geukers is a 3rd year Internal Medicine resident at McMaster University. Dr Matthew Sibbald is an assistant professor and director, Centre for Simulation-Based Learning who practices interventional cardiology at McMaster University. Dr Andrew Gibson is a clinical scholar within the Department of Critical Care. Dr Zahira Khalid is an associate professor at McMaster University and practices General Internal Medicine at St Joseph's Health Care. Dr. Lori Whitehead is a professor of medicine and the director, Internal Medicine Residency Program who practices Respirology and Internal Medicine at McMaster University. Dr. Khalid Azzam is associate professor of Medicine at McMaster University and practices General Internal Medicine at Hamilton Health Sciences. Correspondence may be directed to azzamk@mcmaster.ca

Submitted: June 22, 2017. Accepted: September 15, 2017. Published: March 5, 2018.

\begin{abstract}
\section{Background}

Point-of-Care Ultrasound (PoCUS) is an ultrasound examination performed by the clinician to answer a focused question or guide an invasive procedure. Despite gaining popularity and evidence supporting the use of PoCUS, core Internal Medicine (IM) residency programs in Canada have yet to implement a comprehensive PoCUS curriculum. The objective of this study was to create a formal PoCUS curriculum.
\end{abstract}

\section{Methods}

We conducted a systematic needs assessment with a survey that assessed IM attending and resident comfort, training, and application of PoCUS. We also performed a literature review of selected PoCUS-guided procedures and diagnostics to assess the evidence. A working group analyzed the collected data and designed a graduated 3-year curriculum.

\section{Results}

The needs assessment demonstrated that PoCUS education was both necessary and in high demand. The PoCUS-guided procedures and diagnostics that were identified by the survey to be necessary for IM training were then evaluated by a literature review. Based on the evidence, a progressive 3-year curriculum was created. The working group decided on the method and timing of curriculum delivery.

\section{Conclusion}

McMaster University is the first IM residency program to introduce a graduated 3-year curriculum complete with competency assessment and quality assurance. 


\section{Résumé}

\section{Contexte}

Léchographie au point d'intervention est un examen échographique réalisé par le médecin clinicien pour répondre à une question précise ou guider une intervention effractive. Malgré la popularité grandissante de l'échographie au point d'intervention et les données probantes justifiant son utilisation, les programmes de base de résidence en médecine interne (MI) au Canada nont pas encore mis en place un curriculum complet en échographie au point d'intervention. L’objectif de la présente étude consistait à élaborer un programme officiel en échographie au point d'intervention.

\section{Méthodologie}

Une estimation systématique des besoins a été réalisée au moyen d'un sondage auprès $\mathrm{du}$ personnel enseignant et des résidents en MI. On a ainsi évalué la formation et le degré de confort de ces derniers au regard de l'échographie au point d'intervention, ainsi que l'usage qu'ils font de cette technologie. On a aussi effectué une revue de la littérature portant sur une sélection d'interventions et de diagnostics guidés au moyen de l'échographie au point d'intervention, et ce, dans le but d'évaluer les indications de cette technologie. Un groupe de travail a analysé les données recueillies et a conçu un curriculum séchelonnant sur trois ans.

\section{Résultats}

Lestimation des besoins a démontré que la formation en matière d'échographie au point d'intervention était à la fois nécessaire et très en demande. Le sondage a permis de répertorier un ensemble d'interventions et de diagnostics guidés au moyen de léchographie au point d'intervention qui se devait de faire partie de la formation des résidents en MI. Ces diagnostics et interventions ont par la suite été évalués par une revue de la littérature. À partir des données rassemblées, un curriculum échelonné sur trois ans a été créé. Le groupe de travail a déterminé comment et quand le curriculum devra être appliqué.

\section{Conclusion}

L'Université McMaster est la première université qui dote son programme de résidence en MI d'un curriculum sur léchographie au point d'intervention. Ce curriculum séchelonne sur trois ans et est assorti d'une évaluation des compétences et d'une assurance de la qualité.

Point of Care Ultrasound (PoCUS) is a limited ultrasound examination performed and interpreted by the clinician at the bedside. It is now the standard of care to use ultrasound (US) to guide invasive procedures, such as central line insertion and paracentesis, as it reduces the incidence of complications and number of attempts. ${ }^{1-3}$ In addition, PoCUS can answer focused clinical questions and is used to supplement the physical exam. ${ }^{4,5}$

PoCUS training has become ubiquitous throughout all stages of medical education. A recent study found approximately $50 \%$ of Canadian undergraduate medical programs are teaching US. ${ }^{6}$ PoCUS training is also being incorporated into residency programs. In 2008, US was introduced as a core competency in the Royal College of Physician and Surgeons of Canada Emergency Medicine (EM) Objectives of Training. ${ }^{7,8}$ Since then, formal curricula have been established in EM residency programs. ${ }^{9}$
Internal medicine (IM) is similarly gaining PoCUS interest, with approximately $30-50 \%$ of IM residents using PoCUS. ${ }^{10,11}$ A nationwide survey found that only $25 \%$ of IM programs have formal PoCUS education, with many curricula limited to USguided central venous cannulation. ${ }^{10}$ We recently published that while $27 \%$ of IM residents are using PoCUS to guide clinical decision-making, only $9.7 \%$ of residents have formal PoCUS training. ${ }^{11}$ As with any tool, there is a risk of causing more harm than benefit with inadequate training. Herein, we present our process of curriculum creation and the first 3-year IM PoCUS curriculum.

\section{Methods}

\section{Setting Learning Objectives}

We surveyed all McMaster IM staff and residents on which procedures and diagnostic applications should be included 
in a PoCUS curriculum. ${ }^{11} \mathrm{~A}$ literature search on each PoCUS application was then completed to ensure (1) evidence demonstrated improved patient outcomes or diagnostic accuracy with POCUS compared to standard care, (2) residents could master the skill, and (3) the number of scans required to achieve competency. Learning objectives were identified based on the above exercises.

\section{Steering Committee}

Faculty were recruited along with residents and fellows. These members were divided into subcommittees for each year of the curriculum. Simulation meetings were held five times a year to discuss objectives, curriculum design, resources, competency assessment, program evaluation, and barriers.

\section{Curriculum Overview}

As we have previously published, staff and residents agreed that the four key PoCUS-guided procedures include central line insertion, paracentesis, thoracentesis, and lumbar puncture (LP). These four skills were also identified as a priority for curriculum development in the published document "Expert Consensus on a Canadian Internal Medicine Ultrasound Curriculum." ${ }^{12}$
Diagnostic applications were also assessed. Focused Cardiac Ultrasound (FoCUS) (73\% of staff and $97 \%$ of residents) and Lung Ultrasound (LU) (68\% of staff and $81 \%$ of residents) were the most commonly selected diagnostic applications. ${ }^{11}$ The expert consensus agreed that FoCUS and LU may be applicable to IM training as well. ${ }^{12}$

As such, the following curriculum was proposed: during the residents' Post-Graduate Year-1 (PGY-1), they will learn ultrasound-guided procedures. During PGY-2, residents will be instructed in LU, and the PGY-3s will be taught FoCUS. The specific learning objectives, pre-course material, structure of the curriculum, and competency evaluations will be discussed below.

\section{Year 1 Curriculum Ultrasound-Guided Procedures Learning Objectives}

The specific learning objectives for the PGY-1 PoCUS curriculum are found in Table 1. The selected procedures included USguided Internal Jugular central line insertion, thoracentesis, paracentesis, LP, and arthrocentesis.

Table 1. Specific Learning Objectives for the Post-Graduate Year One Curriculum

Procedure

Internal Jugular Central
Line Insertion

Thoracentesis

Lumber Puncture

Arthrocentesis

\section{Specific Objective}

1.1.1 Identify relevant anatomy for central line insertion

1.1.2 Demonstrate appropriate sterile technique

1.1.3 Demonstrate safe central line insertion technique on partial task trainers

1.1.4 Describe techniques to troubleshoot and/or manage common challenges of central line insertion

1.1.5 Review and discuss indications, contraindications, and complications of the procedure

1.2.1 Identify appropriate anatomy for ultrasound guided thoracentesis

1.2.2 Demonstrate how to safely mark a fluid collection for thoracentesis and estimates the volume of pleural fluid

1.2.3 Demonstrate sterile thoracentesis technique on partial task trainers

1.2.4 Review and discuss indications, contraindications, and complications of procedure

1.2.5 Describe techniques to troubleshoot and/or manage common challenges and complications of lumbar puncture

1.3.1 Identify appropriate anatomy for ultrasound guided paracentesis

1.3.2 Demonstrate safe and sterile ultrasound guided paracentesis technique on partial task trainers

1.3.3 Review and discuss indications, contraindications and complications of the procedure

1.3.4 Describe techniques to troubleshoot and/or manage common challenges and complications of lumbar puncture

1.4.1 Identify anatomy for completion of ultrasound guided lumber puncture

1.4.2 Demonstrate safe ultrasound guided lumber puncture technique on partial task trainers

1.4.3 Review and discuss indications, contraindications, and complications of procedure

1.4.4 Describe techniques to troubleshoot and/or manage common challenges and complications of lumbar puncture

1.5.1 Identify appropriate anatomy for surface land-marking of knee, shoulder, and ankle arthrocentesis

1.5.2 Review and demonstrate arthrocentesis technique on partial task trainers

1.5.3 Review and discuss indications, contraindications and complications of procedure 


\section{Pre-course Material}

Flipped classroom model was applied, and residents were expected to review the pre-course material prior to their arrival. Residents were provided with written manuscripts outlining the procedures and links to videos. For written material, we opted to use the New England Journal of Medicine (NEJM) series. ${ }^{13-17}$ Residents were also directed to watch instructional videos on both the SonoSite website and NEJM to review the US technique for all procedures. . $^{13,14,18-25}$

\section{Delivery of Curriculum}

The curriculum was delivered over two Academic Half Days (AHDs), each lasting three and a half hours. On each day, the residents were divided into six groups of five learners. There were six 30-minute stations: LP, arthrocentesis, two central venous catheterization stations, thoracentesis, and paracentesis. All stations were equipped with a GE ultrasound machine, task trainer, and preceptor (either a senior IM resident or a fellow in critical care, anesthesia, rheumatology, or GIM).

During the first session, participants were encouraged to practice landmarking and perform an abridged version of the procedure. The second session ran six months later. The purpose of the session was to assess and ensure mastery of the procedure, as it was expected that most residents would have had an opportunity to practice during their clinical rotations. The session began with a large group discussion about US-guided procedures and troubleshooting techniques. The preceptors were equipped with procedure-specific checklists (see Appendix A). One resident in the group would complete the entire procedure, including obtaining consent, screening for contraindications, practicing sterile technique, and performing the entire procedure on the partial task trainer. The preceptor in the room would not interrupt the process for teaching or corrections. After the procedure was complete, residents in the group and the preceptor provided feedback. The learners then had time for deliberate practice. This was done at every station, with a different resident being the "lead" every time.

\section{Assessment of Competency and Quality Assurance Outside of the Simulation Setting}

During clinical rotations, PGY-1s were asked to have senior residents, fellows, or attendings observe their US-guided procedures and complete an assessment form. A competencybased assessment form (Appendix A) is kept with each ultrasound machine and was distributed to all residents. The purpose of the direct observation is to provide residents with specific feedback and assess for competency. The residents are expected to compile these forms in their portfolios where they are reviewed annually by the program director.

\section{Evaluation of The Session and Future Directions}

The residents filled out a survey immediately after the session. Based on a likert scale of 1 to 5 ( 1 is strongly disagree and 5 is strongly agree), they agreed to strongly agreed that they were more comfortable performing, with the use of US, central venous cannulaton, arthrocentesis, thoracentesis, and paracentesis after the session. They were neutral to agreed that they felt more comfortable performing LPs. The most common feedback was a desire to have more time to practice. To accommodate this request, we are going to remove the introduction session in the second AHD to maximize the time spent at each station.

\section{Year 2 Curriculum-Lung Ultrasound}

\section{Learning Objectives}

The learning objectives for the PGY-2 PoCUS curriculum are found in Table 2. It was determined that the curriculum would include A and B line profiles, pleural effusions, lung sliding and pneumothorax, consolidation, and the BLUE protocol.

\section{Pre-course Material}

No pre-course reading was assigned.

\section{Delivery of Curriculum}

The curriculum was delivered over two AHDs. The first day was four hours in duration. An initial 60-minute didactic lecture was delivered to the group, which introduced the basics of lung ultrasound including selection of transducer, patient positioning, transducer orientation, land marking, and anatomy. Identification of normal and pathologic lung signatures was reviewed including A and B line profiles, pleural effusions, lung sliding and pneumothorax, consolidation, and the BLUE protocol. The group was then divided in order to ensure close supervision. Half of the residents participated in a one-hour interactive case-based session (approach to shortness of breath using the BLUE protocol). The remaining group was assigned to small groups of three to rotate through four stations. Each station was equipped with a GE ultrasound machine, standardized patient, and preceptor (who was either a GIM, critical care, or EM fellow). At each 15-minute station, residents had the opportunity to practice LU on standardized patients. The residents then switched and participated in the session they had not yet completed. We concluded with a 30-minute full group session in order to review the half-day objectives, answer questions, and conduct a feedback survey.

The second session ran four months later. The purpose of the session was to consolidate image acquisition, image interpretation, and clinical decision-making skills. Groups of three met with a preceptor for two hours and practiced LU on inpatients admitted 
Table 2. Specific Learning Objectives for the Post-graduate Year Two Curriculum

\begin{tabular}{|c|c|}
\hline Topic & Specific Objective \\
\hline Image Acquisition & $\begin{array}{l}\text { 2.1.1 Select the correct transducer } \\
\text { 2.1.2 Appropriately position the patients } \\
\text { 2.1.3 Appropriately handle the transducer } \\
\text { 2.1.4 Landmark the four-points of contact for the hemithorax exam } \\
\text { 2.1.5 Review and discuss indications for lung ultrasound }\end{array}$ \\
\hline Image Interpretation & $\begin{array}{l}\text { 2.2.2 Identify appropriate anatomy including the rib line, bat sign, diaphragm, chest wall, lung, and pleural lines } \\
\text { 2.2.2 Identify A lines and B lines } \\
\text { 2.2.3 Understand A, B and C profiles } \\
\text { 2.2.4 Use of the BLUE protocol }\end{array}$ \\
\hline Pleural Effusions & $\begin{array}{l}\text { 2.3.1 Identify appropriate anatomy surrounding the effusion } \\
\text { 2.3.2 Use appropriate technique to visualize pleural effusions } \\
\text { 2.3.3 Quantitatively estimate the effusion volume } \\
\text { 2.3.4 Describe echogenicity of the effusion (including loculated effusions and the plankton sign) }\end{array}$ \\
\hline Lung Sliding & $\begin{array}{l}\text { 2.4.1 Use the following signs to identify the presence or absence of a pneumothorax: Lung sliding (ants-on-a-log), lung } \\
\text { point, seashore sign or barcode sign, lung pulse, broadening and brightening of the pleural stripe, and absence of } \\
\text { comet tails } \\
\text { 2.4.2 Pitfalls }\end{array}$ \\
\hline
\end{tabular}

to general internal medicine, respirology or the Intensive Care Unit. Patient consent was obtained in advance by the preceptor.

\section{Assessment of Competency and Quality Assurance}

As this was a pilot year, there was no assessment of competency. However, subsequent years will have a LU station at the PGY-2 OSCE. Moreover, residents were provided a USB to store their acquired PoCUS images. We require that all residents collect a minimum of 12 studies, with each of the following pathologic findings being saved for review by a preceptor: A-lines, B-lines, pleural effusion, lung sliding, and consolidation. This is in keeping with the American College of Chest Physician reccomendations. ${ }^{26}$

\section{Evaluation of the Session and Future Direction}

Residents filled out a pre and post-first session quiz and evaluation. Based on a likert scale of 1-4 where $1=$ not useful to $4=$ very useful, residents found the session to be useful to very useful. Of surveyed respondents, $83 \%$ described their comfort with LU as "not comfortable" before the initial AHD. Resident comfort improved after the session, at which point $81 \%$ of residents stated they would be "comfortable performing lung ultrasound with supervision." The most common feedback was a desire to have more time at each station and to have the didactic session available as a recording prior to the session. To improve the PGY-2 curriculum we are going to institute a flipped classroom where the didactic lecture will be recorded for residents to watch prior to their first session and dedicate more time for deliberate practice.

\section{Year 3 Curriculum-Focused Cardiac Ultrasound \\ Learning Objectives}

The specific learning objectives for the PGY-3 FoCUS program are found in Table 3. The two primary learning objective of the FoCUS curriculum are to identify times when it would be appropriate to use FoCUS as an adjunctive tool to the physical examination, and the inherent limitations of FoCUS. The other main objectives of our program were to teach correct image acquisition and trouble shooting of the 5 basic views (parasternal-long axis, parasternal short axis, apical-4 chamber, subcostal 4-chamber and subcostal inferior vena cava views), and to identify gross pathology (right ventricular [RV] dysfunction, left ventricular [LV] systolic dysfunction, pericardial effusions, tamponade, signs of pulmonary embolism, and cardiac arrest).

\section{Pre-course Material}

We applied the flipped classroom model. There were 4 components of pre-course activity to complete: readings, videos, an interactive e-learning module, and worksheets.

The reading assignment were from Soni et al's textbook Point-of-Care Ultrasound..$^{27}$ This book was available through our library. Residents were asked to read six chapters including 
Table 3. Specific Learning Objectives for the PGY-3 Curriculum

Topic

Perform a Goal-Directed, Focused Cardiac Ultrasound

Left Ventricular Systolic Dysfunction

Right Ventricular Dysfunction

Appropriate Use of Cardiac

Ultrasound to Guide Management

Cardiac Arrest

Pericardial Effusion

\section{Specific Objective}

3.1.1 Properly position the patient to optimize views

3.1.2 Acquire and interpret images which demonstrate a) Parasternal long-axis, b) Parasternal short-axis with mix-ventricular level view, c) Apical 4-chamber view, d) Subcostal 4-chamber view, e) Subcostal inferior vena cava view

3.1.3 Identify all relevant anatomy

3.1.4 Discuss common pitfalls in the bedside cardiac ultrasound exam

3.1.5 Identify clinical situations where cardiac ultrasound and formal echocardiography is most appropriate

3.2.1 Acquire and interpret diagnostic quality images that assess LV systolic function (including examination of endocardial excursion, anterior mitral leaflet movement, myocardial thickening, gross detection of regional wall motion abnormalities, and assessment for hyperdynamic)

3.2.2 Consider the role of bedside cardiac ultrasound compared to other components of the physical exam for the diagnosis of left sided heart failure

3.3.1 Acquire and interpret diagnostic quality images for RV systolic function (including examination of RV size, shape, wall thickness, tricuspid annular plane systolic excursion, septal movement, and gross wall motion abnormalities)

3.3.2 Consider the relationship between RV dysfunction, $\mathrm{PE}$, and heart failure

3.3.3 Understand the findings of a PE on bedside cardiac ultrasound: RV/LV $>1$, free floating thrombus, decreased RV function

3.3.4 Consider the differential of RV enlargement

3.4.1 Volume status

3.4.2 Hypotension or shock

3.4.3 Cardiac arrest

3.5.1 Be able to identify organized cardiac contractility to help distinguish between asystole, PEA, and pseudo-PEA.

3.5.2 Understand what views should be used and discuss appropriate timing during a code blue

3.5.3 Understand how one can determine the cause of cardiac arrest

3.6.1 Understand which views to use to correctly diagnose pericardial effusions

3.6.2 Understand how to differentiate between pericardial effusions, pleural effusions, peritoneal fluid, and epicardial fat pad

3.6.3 Understand the relationship between the presence of pericardial fluid and tamponade

3.6.4 Understand the role of bedside cardiac ultrasound compared to other components of the physical exam, which supports the diagnosis of tamponade

3.6.5 Understand the limitations of cardiac ultrasound in diagnosis of tamponade
Chapter 12 "Overview," Chapter 13 "Technique," Chapter 14 "Left Ventricular Function," Chapter 15 "Right Ventricular Function," Chapter 17 "Pericardial Effusions," and Chapter 18 "Inferior Vena Cava." This provided the background knowledge and introduction to FoCUS.

Participants were directed to watch a series of videos from SonoSite and EchoVTutorials ${ }^{28-35}$ where troubleshooting image acquisition was demonstrated in real time.

The third component required participants to apply their knowledge. Residents were asked to work through the Toronto General Hospital Department of Anesthesia Perioperative Interactive
Education Focused Cardiac Ultrasound virtual transthoracic echocardiography webpage. ${ }^{36}$ It provided an opportunity for learners to interact with the FoCUS views, manipulate the heart, add or remove anatomy labels, and gain a deeper understanding of FoCUS. It also explained pathology such as hypovolemia, RV dysfunction, LV dysfunction, and tamponade.

The final component required participants to complete a workbook. The first task asked participants to label the cardiac view depicted, describe how to position the patient, as well as the position and orientation of the US probe. The second worksheet asked participants to evaluate pre-existing echo 
videos and comment on the view, the position and quality of the image, and how to correct existing errors. The third component asked participants to comment on the RV and LV function of ten different echo videos,

\section{Delivery of Curriculum}

The curriculum was offered over two days. The first day was scheduled over two hours. Half of the PGY-3 class was randomly selected to participate to determine feasibility. In total, 12 residents were divided into three groups. There were three 30 -minute stations. Station one was a Vimedix simulator (a high-fidelity cardiac ultrasound simulator). Participants were required to find each of the five cardiac views and then identify pathology, including left atrial thrombi, tamponade, LV free wall rupture, and regional wall motion abnormalities. For the second station, groups were divided into two with each subgroup entering a different room with a cardiologist, standardized patient, and ultrasound machine. Participants were asked to demonstrate each of the five views under the guidance of the preceptor. The final station involved case discussions. The cases were created to stimulate discussion about the appropriate use of FoCUS in diagnostic dilemmas such as heart failure, fluid resuscitation, and cardiac arrest.

The second day required residents to organize a small group session with a cardiologist in a hospital. FoCUS exams were performed on patients with and without pathology to consolidate learning.

\section{Assessment of Competency and Quality Assurance}

As this was a pilot year, there was no assessment of competency. However, subsequent years will have a FoCUS station at the PGY-3 OSCE. Residents were provided a USB to record their images, and it will be expected that they complete a minimum of ten studies, to be reviewed by a preceptor. A total of 50 scans will be required, as recommended by the American College of Chest Physicians. ${ }^{26}$

\section{Evaluation of the Session and Future Directions}

The residents completed a survey immediately after the session. Based on a likert scale of 1 to 5 where the residents were asked to rate the effectiveness of the program (1 correlated to no change in their ability to perform FoCUS and 5 correlating with optimal improvement in their skills), $82 \%(9 / 11)$ rated the session a 5 and $2 / 11$ residents rated it a 4 . The most common constructive feedback was a desire to have more time at each station. To amend this, we are going to recruit more preceptors to add additional stations in future years.

\section{Discussion}

PoCUS has the potential to be an extremely useful tool. However, there is a concern that PoCUS studies performed by inexperienced practitioners may result in errors, increased investigations, and negatively impact patient outcomes. The Canadian Association of Radiologists 2013 position statement on PoCUS echoed this concern. ${ }^{37}$ We agree that PoCUS is a tool that deserves respect. Given that US machines are becoming more available throughout hospitals, and trainees are using them without formal training to make clinical judgments, we felt it was crucial to introduce PoCUS training in our residency curriculum. As such, we present the first comprehensive, 3-year graduated PoCUS curriculum introduced to a Canadian IM residency program.

As this is the first PoCUS curriculum, there were numerous challenges including faculty development, cost, and assessment of competence. Based on our survey, only 33\% of GIM staff have some formal training in the diagnostic applications of PoCUS and $40 \%$ have formal training in PoCUS guided procedures. ${ }^{11}$ This led to the involvement of subspecialty colleagues. For instance, the LU course was taught by intensivists, respirologists, and critical care fellows while cardiologists taught out FoCUS curriculum. GIM staff have been encouraged to pursue further training through PoCUS continuing professional development courses.

The cost of the program was minimal. The IM Residency Program financed renting the simulation centre, hiring standardized patients, and buying USBs. The McMaster University Centre for Simulation-Based Learning provided the task trainers and two US machines free of cost. The McMaster cardiology fellowshiptraining program supplied the Vimedex, and GE provided us with additional ultrasound machines free of cost. Please see Appendix A for a breakdown of costs.

As stated above, PoCUS is a powerful tool that requires appropriate training and ongoing quality assurance. We believe we have created a solid platform for quality assurance within the PGY-1 procedural curriculum. Our goal moving forward is to create competency-based evaluations for the PGY-2 and PGY-3 program. The process of evaluation will be three fold. Residents have been provided with USBs to capture and review their images with a staff. Second, we are going to host citywide PoCUS rounds every 3 months where a resident will present a case and accompanying images. Finally, we plan to integrate a PoCUS acquisition and interpretation station into the second and third year annual OSCE.

\section{Conclusion}

In conclusion, McMaster University is the first IM residency program to introduce a graduated 3-year curriculum complete with competency assessment and quality assurance. This work 
can provide a blueprint for other IM programs looking to develop PoCUS curriculum.

\section{Disclosure}

The authors have no conflicts of interest or funding to disclose.

\section{References}

1. Brass P, Hellmich M, Kolodziej L, et al. Ultrasound guidance versus anatomical landmarks for subclavian or femoral vein catheterization. In: Brass P, ed. Cochrane Database of Systematic Reviews. Vol 1. Chichester, UK: John Wiley \& Sons, Ltd; 2015:CD011447.

2. Randolph AG, Cook DJ, Gonzales CA, et al. Ultrasound guidance for placement of central venous catheters: a meta-analysis of the literature. Crit Care Med 1996;24(12):2053-58.

3. Patel PA, Ernst FR, Gunnarsson CL. Evaluation of hospital complications and costs associated with using ultrasound guidance during abdominal paracentesis procedures. J Med Econ 2012;15(1):1-7.

4. Kircher BJ, Himelman RB, Schiller NB. Noninvasive estimation of right atrial pressure from the inspiratory collapse of the inferior vena cava. Am J Cardiol 1990;66(4):493-96.

5. Nagueh SF, Kopelen HA, Zoghbi WA. Relation of mean right atrial pressure to echocardiographic and Doppler parameters of right atrial and right ventricular function. Circulation 1996;93(6):1160-69.

6. Steinmetz P, Dobrescu O, Oleskevich S, et al. Bedside ultrasound education in Canadian medical schools: A national survey. Can Med Educ J 2016;7(1):e78-86.

7. Kim DJ, Theoret J, Liao MM, et al. The current state of ultrasound training in canadian emergency medicine programs: perspectives from program directors. Acad Emerg Med 2012;19(9):E1073-E1078.

8. Royal College of Physicians and Surgeons of Canada. Objectives of training in the specilty of emergency medicine 2014. 2014. Available at: http://www. royalcollege.ca/cs/groups/public/documents/document/y2vk/mdaw/ edisp/ tztest3rcpsced000895.pdf.

9. Boulger C, Adams DZ, Hughes D, et al. Longitudinal ultrasound education track curriculum implemented within an emergency medicine residency program. J Ultrasound Med February 2017.

10. Ailon J, Mourad O, Nadjafi M, et al. Point-of-care ultrasound as a competency for general internists: a survey of internal medicine training programs in Canada. Can Med Educ J 2016;7(2):e51-e69.

11. Lewis K, McConnell M, Azzam K. A systematic needs assessment for point of care ultrasound in internal medicine residency training programs. Can J Gen Intern Med 2017;12(2):23-30.

12. Arishenkoff S, Blouw M, Card S, et al. Expert Consensus on a Canadian internal medicine ultrasound curriculum. Can J Gen Intern Med 2014;9(3).

13. Thomsen TW, DeLaPena J, Setnik GS. Thoracentesis. N Engl J Med 2006;355(15):e16.

14. Thomsen TW, Shaffer RW, White B, et al. Paracentesis. N Engl J Med. 2006;355(19):e21.

15. Thomsen TW, Shen S, Shaffer RW, et al. Videos in clinical medicine. Arthrocentesis of the knee. N Engl J Med 2006;354(19):e19.

16. Ortega R, Song M, Hansen CJ, et al. Videos in clinical medicine. Ultrasoundguided internal jugular vein cannulation. N Engl J Med 2010;362(16):e57.

17. Ellenby MS, Tegtmeyer K, Lai S, et al. Videos in clinical medicine. Lumbar puncture. N Engl J Med 2006;355(13):e12.
18. Ortega R, Song M, Hansen CJ, et al. Ultrasound-guided internal jugular vein cannulation. N Engl J Med 2010;362(16):e57.

19. Ellenby MS, Tegtmeyer K, Lai S, et al. Lumbar Puncture. N Engl J Med 2006;355(13):e12.

20. Thomsen TW, Shen S, Shaffer RW, et al. Arthrocentesis of the Knee. N Engl J Med 2006;354(19):e19.

21. FujiFilm SonoSite. 3D How To: Lateral Knee Injection | SonoSite. Available at: https://www.sonosite.com/media-library/3d-how-lateral-knee-injection.

22. FujiFilm SonoSite. 3D How To: IJV catheter insertion (Transverse)

| SonoSite. Available at: https://www.sonosite.com/ca/ media-library/3d-how-ijv-catheter-insertion-transverse.

23. FujiFilm SonoSite. 3D how to: ultrasound guided paracentesis | SonoSite. Available at: https://www.sonosite.com/ca/ media-library/3d-how-ultrasound-guided-paracentesis.

24. FujiFilm SonoSite. 3D how to: lumbar puncture procedure. SonoSite. Available at: https://www.sonosite.com/ca/ media-library/3d-how-lumbar-puncture-procedure.

25. FujiFilm SonoSite. 3D how to: ultrasound guided thoracentesis. SonoSite. Available at: https://www.sonosite.com/ca/ media-library/3d-how-ultrasound-guided-thoracentesis.

26. American College of Chest Physicians. Critical care ultrasonography certificate of completion program - American College of Chest Physicians. Available at: http://www.chestnet.org/Education/Advanced-Clinical-Training/ Certificate-of-Completion-Program/Critical-Care-Ultrasonography.

27. Soni N, Arntfield R, Kory P. Point-of-care ultrasound. Philadephia, PA: Elsevier Saunders; 2015.

28. EchoVTorials. Intro to Echo Apical 4 Chamber View. mov - YouTube. Available at: https://www.youtube.com/ watch? $v=$ XiTRNaU8_NA\&feature=BFa\&list=PL64A5A87B5044A52D.

29. EchoVTorials. Intro to echo parasternal views.mov - YouTube. Available at: https://www.youtube.com/ watch $? \mathrm{v}=\mathrm{aX}$ worpaecjM\&feature $=\mathrm{BFa} \&$ list $=$ PL64A5A87B5044A52D.

30. SonoSite. How to: focused echo - subcostal view with ultrasound - YouTube. Available at: https://www.youtube.com/watch?v=Mkc6tUVRgKo.

31. SonoSite. Cardiac ultrasound - apical view - SonoSite, Inc. - YouTube. Available at: https://www.youtube.com/ watch $? \mathrm{v}=4 \mathrm{vBJoWP}-\mathrm{zBM} \&$ feature $=$ related.

32. SonoSite. Cardiac ultrasound - parasternal short axis - SonoSite, Inc. YouTube. Available at: https://www.youtube.com/watch?v=B731sgCuZU4.

33. SonoSite. Cardiac ultrasound - parasternal long axis - part 2 - SonoSite, Inc. YouTube. Available at: https://www.youtube.com/watch?v=uciGL4TaoaA.

34. SonoSite. Cardiac ultrasound - parasternal long axis - part 1 - SonoSite, Inc. YouTube. Available at: https://www.youtube.com/watch?v=H_3V9xlDMA0.

35. EchoVTorial. Echocardiography How to Find a Window. mov - YouTube. Available at: https://www.youtube.com/ watch?list $=$ PL64A5A87B5044A52D\&v=482CdbvapBU\&feature $=$ player embedded.

36. Toronto General Hospital Department of Anesthesia. Focused cardiac ultrasound transthoracic echocardiography TTE PIE Toronto FOCUS FATE standard views tamponade hypovolemia ventricular dysfunction. Available at: http://pie.med.utoronto.ca/TTE/TTE_content/focus.html\#introduction.

37. Canadian Association of Radiologists. Position statement on the use of point of care ultrasound. 2013. Available at: http://www.car.ca/uploads/standards guidelines/point_of_care_ultrasound_position_statement_20140527.pdf. 